\title{
Editorial - The misassumptions about contributions
}

In the past year, a large portion of new submissions to the Journal of Research in Interactive Marketing have been desk rejected or unsubmitted. In addition, a large number of manuscripts have been rejected after a few rounds of revision. Most decision letters for those rejected manuscripts included a common comment: "your manuscript does not demonstrate adequate contributions to the extant literature."

While most authors know the importance of making contributions when submitting a paper to an academic journal, unfortunately, a number of authors might make incorrect assumptions regarding how to make a contribution. The following are some common and implicit assumptions that I have observed from the submissions to JRIM.

\section{Grand model makes a big contribution}

Quite a number of papers start with a very comprehensive conceptual model or framework with many predicting paths connecting many antecedents, mediators (and serial mediators) and consequences. As a result, a long string of hypotheses is developed to connect all variables in the framework with little solid theoretical foundation and conceptual development. It is not uncommon that the majority of those hypotheses are developed just because they are parts of the connecting paths in the model, not because they are actually conceptually innovative or theoretically important. Such hypotheses are largely intuitive or common knowledge that we have already known from the extant literature (such as "consumer attitude leads to purchase intention," "satisfaction leads to loyalty" and "brand trust increases purchasing behavior"). Since we already know the outcome before we test the relationship between such variables, what is the purpose for which we conduct such an empirical study? Simply summarizing or "integrating" existing knowledge and findings from different research streams into a grand model does not add much value to knowledge development.

\section{More hypotheses make more contributions}

Some authors might think that a paper with fewer hypotheses does not sufficiently show a contribution. As such, they add many unnecessary predictors or outcome variables that are nothing novel and interesting in a long list of hypotheses. Some of those manuscripts attempt to add to the number of hypotheses by dividing one construct into several dimensions and writing one hypothesis for each dimension. While it might be possible that each dimension has a unique prediction on certain outcomes or can be predicted by different antecedents with strong theoretical justification, however, what is the purpose of developing a string of hypotheses based on the same conceptualization and prediction for each dimension (i.e. X1, X2 and X3 all positively influence Y)? Many such manuscripts are apparently data driven, and the authors simply include more variables in a statistical test beforehand to see "what will have happen" and then write the hypotheses retroactively. Such purely data-driven hypotheses are often meaningless because of the lack of a theoretical foundation and conceptual explanation. In many cases, it would be much better to develop a couple of interesting and insightful hypotheses than a dozen intuitive hypotheses that are too boring to read. Sometimes a potentially interesting idea or finding could be buried in a long string of common sense hypotheses, thus hurting the potential contribution. Less is more!

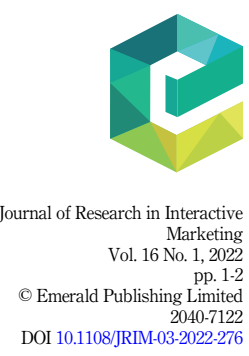


JRIM

16,1

It is also quite common to see some manuscripts attempt to "fill a literature gap" by stating that "This topic has not been studied in the literature" or "this is the first empirical study conducted in $\mathrm{x}$ country or in y industry [...]." A phenomenon or topic that has never been examined before does not necessarily indicate a research gap, and a first attempt to investigate a research topic in a specific context does not always mean a contribution. It could be a trivial or meaningless issue, unless you demonstrate its importance and explain why it should not be ignored. An interesting study is not evaluated by whether it has been studied; it should indicate something important but overlooked (e.g. an important intervening variable, a boundary condition or resolving controversial and contrasting findings), adding new value, new insight and many times counterintuitive and beyond conventional wisdom. According to Davis (1971, p. 313), an interesting study would demonstrate "what seems to be $\mathrm{X}$ is in reality non-X or what is accepted as $\mathrm{X}$ is actually a non-X." Therefore, a study can be considered "innovative" or "novel" must demonstrate adding something important and insightful to theoretical advancement, practical guidelines or stimulate future research inquiry.

\section{Applying a famous theory adds a contribution}

Some manuscripts attempt to apply well-established theories to any research topic, e.g. an interactive marketing phenomenon, but do not show how such theories are relevant to the topics being investigated, although the theories are applied in new fields (e.g. voice assistance technology, virtual reality or e-WOM). In other words, such studies follow the traditional paradigm of the established model with common variables specified by the model (e.g. the theory of planned behavior, new product adoption and diffusion, the technology acceptance model, etc.) but shed little light on the particular phenomenon being examined. Eventually, such studies just add one more repetition to hundreds of existing studies to "prove" the theory. Interactive marketing is a relatively new field but also one of the fastest growth areas due to advancements in new technology, participatory culture, platform revolution and social media and mobile marketing practices (Wang, 2021). The field demands new theoretical advancements and knowledge development. There is great room for making new contributions to the field by addressing new theoretical and practical issues in the ever-changing interactive marketing landscape. It is essential for manuscripts submitted to JRIM to demonstrate that it adequately adds new knowledge to the interactive marketing literature by bringing new understanding of interactive phenomena through theoretical perspectives that are more relevant.

As the editor of Journal of Research in Interactive Marketing, I am looking forward to more innovative, interesting and insightful research papers that make real contribution to knowledge advancement in the field of interactive marketing.

\section{Cheng Lu Wang}

\section{References}

Davis, M.S. (1971), "That's interesting! Towards a phenomenology of sociology and a sociology of phenomenology", Philosophy of the Social Sciences, Vol. 1 No. 2, pp. 309-344.

Wang, C.L. (2021), "New frontiers and future directions in interactive marketing: inaugural editorial", Journal of Research in Interactive Marketing, Vol. 15 No. 1, pp. 1-9. 